\title{
Eye Muscle Weakness
}

National Cancer Institute

\section{Source}

National Cancer Institute. Eye Muscle Weakness. NCI Thesaurus. Code C78291.

A reduction in the strength of the eye muscles. 